J. Sustain. Wireless Syst.

Vol.01/ No. 03

Pages: 189-197

http://irojournals.com/irosws/

DOI: https://doi.org/10.36548/jsws.2019.3.005

\title{
5G INTEGRATED MOBILE SATELLITE SERVICE WITH HIGH ENERGY EFFICIENCY
}

\author{
Joy Iong-Zong Chen, \\ Professor, \\ Dept. Electrical Engineering of Da-Yeh University, Taiwan, ROC. \\ Email: jchen@mail.dyu.edu.tw
}

\begin{abstract}
Communication technology is moving towards its next generation network and 5G implementation. 5G provides high resource efficiency, continuity, reduced power consumption and increased resilience. This technology is integrated with mobile satellite service (MSS). This new architecture improves the commercial value proposition of the network, provide optimal performance and energy efficiency. It improvises the ubiquity, mobility, broadcast and security features of the existing communication system. It also provides an efficient broadband connectivity in moving platforms and good broadcast services. The long propagation delay of MSS is also addressed in this paper. We also the meet the increased backhaul demands thereby saving physical sites on ground.
\end{abstract}

Keywords: 5G, Mobile Satellite Service, Energy Efficiency, Wireless Communication, wireless network

\section{INTRODUCTION}

There has been a rapid revolution in mobile cellular communication system along a set of standards termed as Generations. There generations evolved from 1G analogue technology, 2G GSM (Global System for Mobile) technology, 3G IMT-2000 (International Mobile Telecommunications-2000) technology to the current 4G LTE (Long-Term Evolution) technology [4]. Since the recent past, research has been focused towards developing 5G technology. The 5GPPP (5G Infrastructure Public Private Partnership) was established to perform research and provide solutions, standards, technologies and architectures for the next generation communication infrastructure. $5 \mathrm{G}$ offers better reliability, capacity and better broadband width when compared to $4 \mathrm{G}$ network. It offers fast data transfer that matches with the high speed fibre optic network. $5 \mathrm{G}$ offers a very high frequency of up to $20 \mathrm{Gbps}$.

Along with these technologies, satellite communication systems has also been evolving independent of terrene entity. Echo 1, telstar, syncom, Inmarsat and so on have been serving as communication satellites since the 1960s. Due to the unique characteristics of mobile satellite systems and the capability to establish reliable communication network even in complex scenarios like during transportation, during emergency and in remote 
J. Sustain. Wireless Syst.

Vol.01/ No. 03

Pages: 189-197

http://irojournals.com/irosws/

DOI: https://doi.org/10.36548/jsws.2019.3.005

areas, it play a substantial role in the next generation wireless communication. The NetWorld2020 ETP (European Technology Platform) also works on analysing the implementation methods of satellites in 5G.

Nine satellites are operated by Inmarsat in the geostationary orbit. These satellites serve over $90 \%$ of the long-distance travellers at sea and on cruise ships [6]. The mobile traffic through sea is increasing annually at a rate of $40 \%$. Similarly, aeronautical satellite communication services is also made available across the globe. This assists the long distance business travellers to cater to their business needs even during travel. Inmarsat is in operation across the Atlantic, Indian and Pacific Ocean region and consumes a power of 25-39dBW. Other than the limited spectrum constraint, this system provides endless number of services to the travellers.

The comparison between various generations of mobile technologies with respect to bandwidth, services, switching and other major factors is as represented in table 1. 1G was introduced by Telecom aka Telstra in the year 1987. It had some major drawbacks like call drops, poor voice quality and phone with bad battery life. It was later upgraded to $2 \mathrm{G}$ with CDMA (Code Division Multiple Access) and GSM that allowed making conference calls, holding calls, internal roaming and so on. 3G introduced the world to smartphone technology and low cost data transmission. It used UMTS (Universal Mobile Telecommunication System) architecture. 4G offered high speed and advanced services like mobile TV, cloud computing, video conferencing and so on.

Table 1: Comparison of mobile technologies from $1 \mathrm{G}$ to $5 \mathrm{G}$

\begin{tabular}{|c|c|c|c|c|c|}
\hline Technology & $1 G$ & $2 \mathrm{G}$ & $3 G$ & $4 \mathrm{G}$ & $5 G$ \\
\hline Deployment & $1970 / 1984$ & $1980 / 1999$ & $1990 / 2002$ & $2000 / 2010$ & $2014 / 2015$ \\
\hline Bandwidth & $2 \mathrm{kbps}$ & 14-64kbps & $2 \mathrm{mbps}$ & $200 \mathrm{mbps}$ & $>1 g b p s$ \\
\hline Technology & $\begin{array}{l}\text { Analog } \\
\text { cellular }\end{array}$ & Digital cellular & $\begin{array}{l}\text { Broadband width/ } \\
\text { cdma/ ip technology }\end{array}$ & & 4G+WWWW \\
\hline Services & $\begin{array}{l}\text { Mobile } \\
\text { telephony }\end{array}$ & $\begin{array}{l}\text { Digital voice, } \\
\text { short messaging }\end{array}$ & $\begin{array}{l}\text { Integrated high } \\
\text { quality audio, video } \\
\text { and data }\end{array}$ & $\begin{array}{l}\text { Dynamic } \\
\text { information access, } \\
\text { variable devices }\end{array}$ & $\begin{array}{l}\text { Dynamic information } \\
\text { access, variable devices } \\
\text { with Al capabilities }\end{array}$ \\
\hline Multiplexing & FDMA & TDMA/CDMA & CDMA & CDMA & CDMA \\
\hline Switching & Circuit & $\begin{array}{l}\text { Circuit/circuit for } \\
\text { access network \& } \\
\text { air interface }\end{array}$ & $\begin{array}{l}\text { Packet except for air } \\
\text { interface }\end{array}$ & All packet & All packet \\
\hline Core Network & PSTN & PSTN & Packet network & Internet & Internet \\
\hline Handoff & Horizontal & Horizontal & Horizontal & $\begin{array}{l}\text { Horizontal \& } \\
\text { vertical }\end{array}$ & Horizontal \& vertical \\
\hline
\end{tabular}


J. Sustain. Wireless Syst.

Vol.01/ No. 03

Pages: 189-197

http://irojournals.com/irosws/

DOI: https://doi.org/10.36548/jsws.2019.3.005

\section{EXISTING LITERATURE}

Research has been in progress for creating advancements in communication systems since times immemorial. Konstantinos et al., [1] presented a paper on 5G integration with satellite-terrestrial networks using the SaT5G approach for enhancing mobile broadband services. It reviews the Sat5G objectives, concepts and research pillars along with the use cases, selection methodologies and scenarios that initiates the architecture design and integrated network topology. Yonghwa et al., [2] performed an evaluation of mobile satellite communication that deals with high frequencies. It performs a comparative analysis of conventional satellite models depending on various altitudes fog Low Earth Orbit (LEO), Medium Earth Orbit (MEO), Geostationary Orbit (GEO) and High-altitude platforms (HAP).

Software Defined Satellite communication based on 5G - H2020 SaT5G is studied in [3] by Hamzeh Khalili et al. The paper discuses certain potential use cases for integration of $5 \mathrm{G}$ terrestrial networks along with next generation satellite systems. It also provides a brief overview of technologies like Massive Machine-Type Communication (mMTC), Ultra Reliable and Low Latency Communications (URLLC) and Enhanced Mobile Broadband (eMBB). Figure 1 represents a basic mobile satellite communication system.

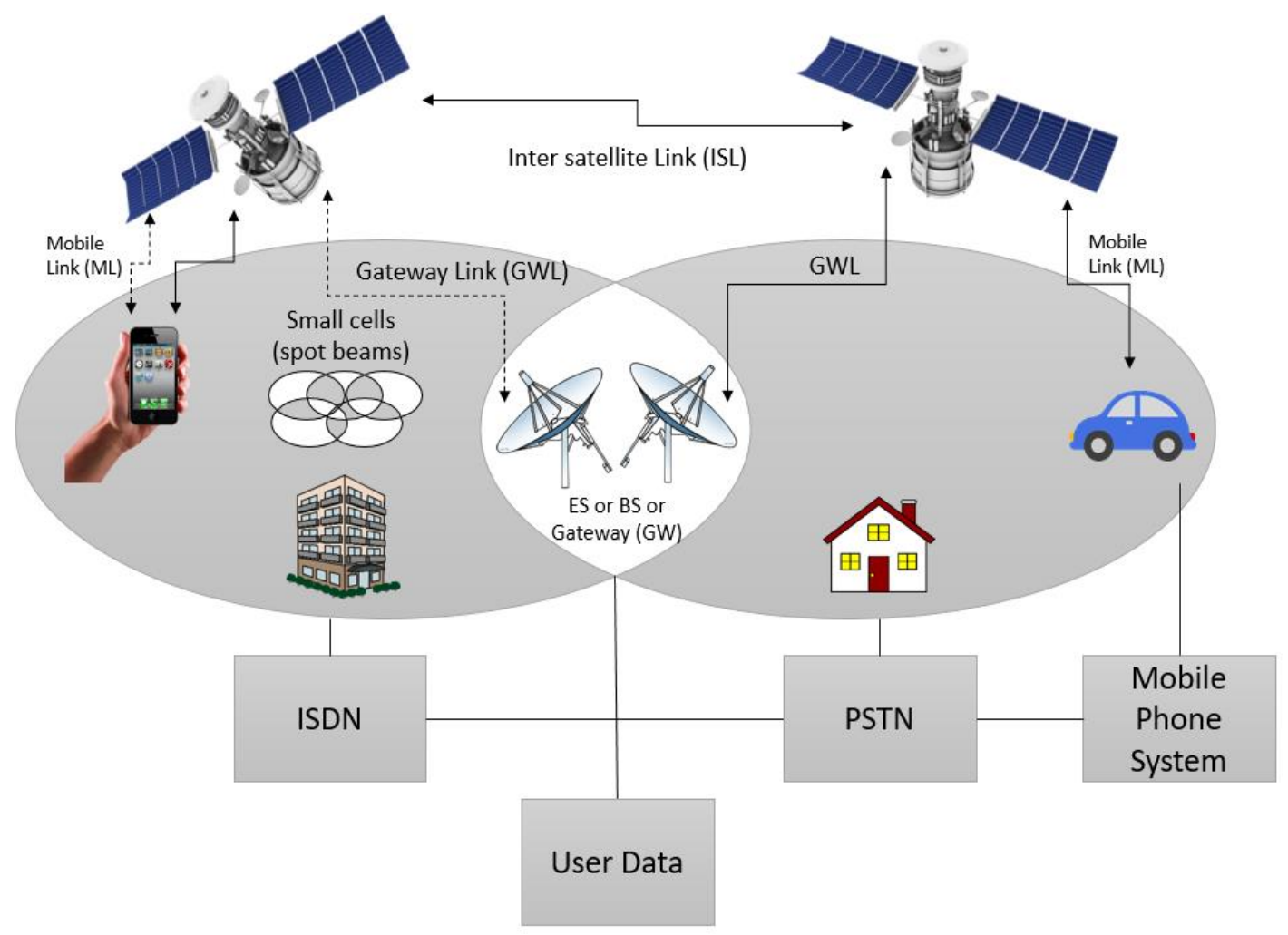

ISSN: 2582-3167 (online) 
J. Sustain. Wireless Syst.

Vol.01/ No. 03

Pages: 189-197

http://irojournals.com/irosws/

DOI: https://doi.org/10.36548/jsws.2019.3.005

Figure 1: Mobile Satellite Communication System

Stavroula Vassaki et al., [5] presented an optimal system satisfying the quality of service constraints for allocation of power in mobile satellite systems and services. Min Jia et al., [7] proposed a hybrid satellite communication system providing broadband services with cognitive radio and 5G technology. This cognitive radio based satellite-terrestrial communication system uses spectrum sensing to design a space segment. They system also involves an algorithm that works on cooperative spectrum sensing technology. Ka Ming et al., [8] developed patch antenna that is circularly polarised for the purpose of high speed communication in 5G technology. This antenna covers the azimuth range and a wide elevation angle.

Jiazin Zhang et al. [9] applied software defined features for developing a satellite terrestrial network that is hybrid and provides end to end coverage with improved energy efficiency and performance metrics. This enables reduced overhead cost. Figure 2 represents the applications of mobile satellite in urban, rural and remote areas as obtained from Inmarsat. The applications vary widely from public mobile telephone services to government defence services.

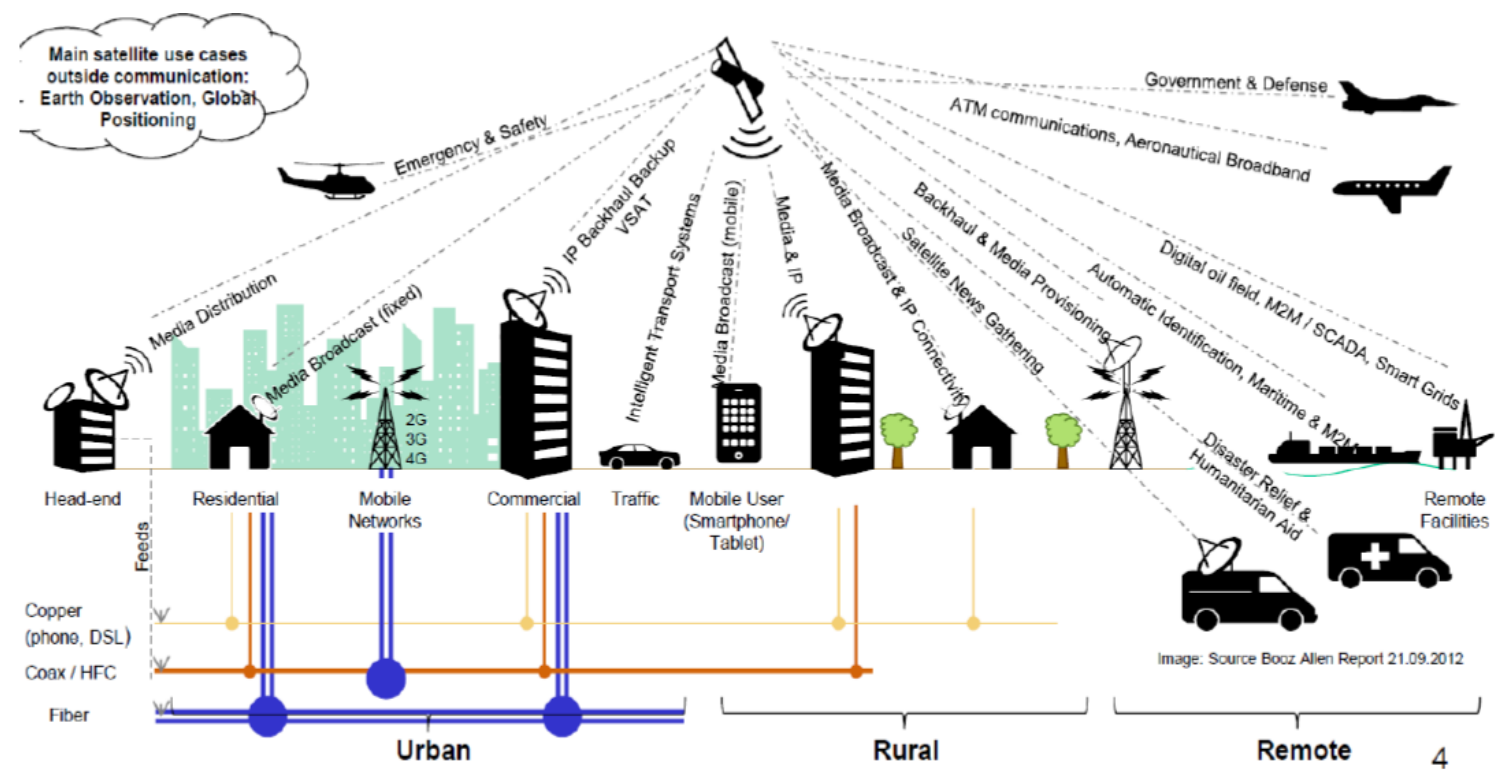

Figure 2: Applications of mobile satellite based on data from Inmarsat 
J. Sustain. Wireless Syst.

Vol.01/ No. 03

Pages: 189-197

http://irojournals.com/irosws/

DOI: https://doi.org/10.36548/jsws.2019.3.005

Korany et al. [10] designed an antenna array package that is circularly polarised with dual-band for 5G communication. It also offers capabilities of beam-steering and offers dual resonance with an axial ratio of $<3 \mathrm{~dB}$ and gain of $>7.5 \mathrm{~dB}$.

\section{PROPOSED WORK}

For providing energy efficiency, it is essential to assign ideal power to the system. Adaptive Multi-Phase Shift Keying and Multiple Quadrature Amplitude Modulation techniques are used for this purpose along with Bit Error Rate based approximation. The service rate of data transfer using these techniques is given by

$$
R_{S}\left(y_{s}, B E R, P\left(z, y_{s}\right)\right)=\frac{T_{f} B}{c} \log _{2}\left[d-\frac{b y_{s}}{\ln \left(\frac{B E R}{a}\right)} P\left(z, y_{s}\right)\right]
$$

Where $\mathrm{a}, \mathrm{b}, \mathrm{c}, \mathrm{d}$ are constants based on the type of modulation and approximation, $\mathrm{y}_{\mathrm{s}}[\mathrm{n}]$ represents the signal to noise ratio, $\mathrm{z}$ denotes QoS parameter, $\mathrm{B}$ is the total spectral bandwidth, $\mathrm{T}_{\mathrm{f}}$ is the duration required for organizing the upper layer packets into frames and $\mathrm{P}\left(\mathrm{z}, \mathrm{y}_{\mathrm{s}}\right)$ represents the power allocation policy.

The ideal power assignment can be expressed as follows

$$
P\left(z, y_{s}\right)=\left\{\begin{array}{c}
A,\left(\frac{d}{y_{s}}-\frac{1}{y_{\min }^{\frac{c}{c+x z}} y_{s}^{\frac{x z}{c-x z}}}\right), \text { for } y_{s} \geq y_{\min } \\
0, \text { else }
\end{array}\right.
$$

Where, $A=\ln \frac{\frac{B E R}{a}}{b}, x=T_{f} B / \ln 2$ and the minimum SNR is given by $\mathrm{y}_{\min }$.

An optimal capacitance can also be provided in order to improve the energy efficiency given by the equation

$$
E_{c}(z)=-\frac{1}{Z} \ln \left(\varepsilon\left\{e^{-z R[n]}\right\}\right)
$$

\section{RESULT}

ISSN: 2582-3167 (online) 
J. Sustain. Wireless Syst.

Vol.01/ No. 03

Pages: 189-197

http://irojournals.com/irosws/

DOI: https://doi.org/10.36548/jsws.2019.3.005

\section{Capacitance, elevation and QoS comparison}

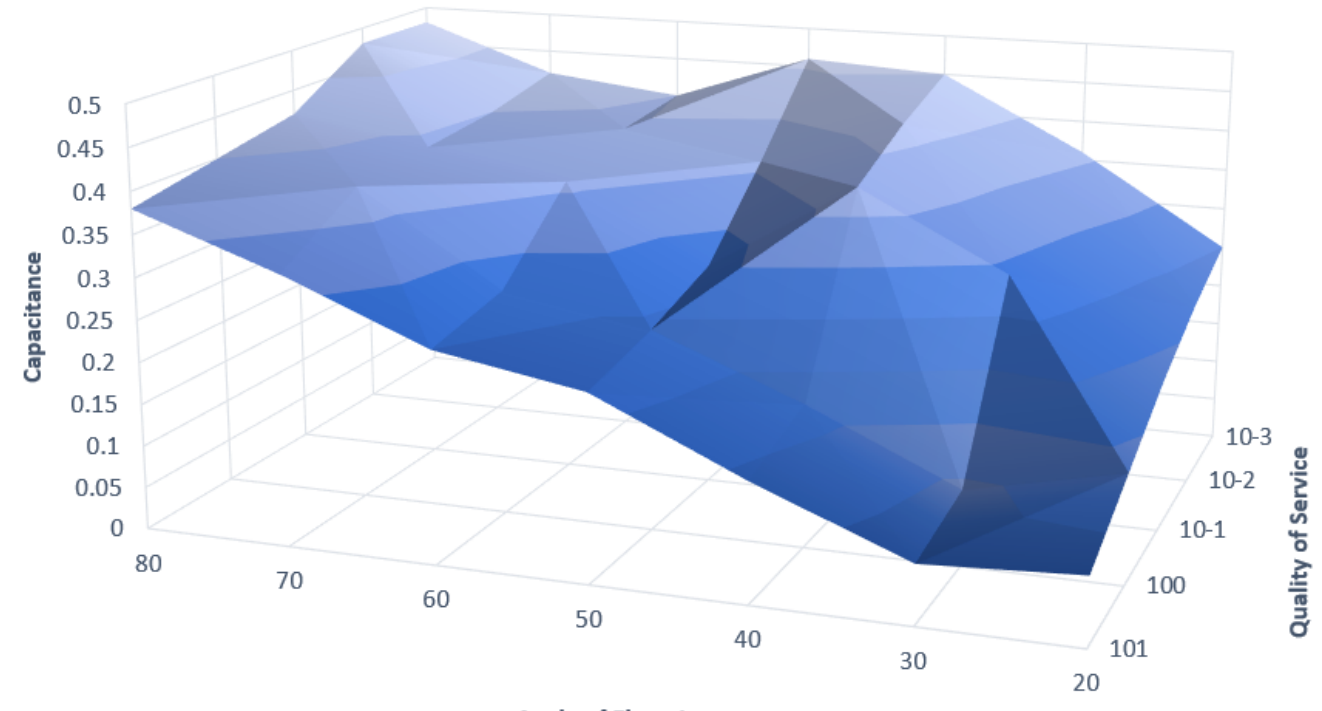

Angle of Elevation

Figure 3: Capacitance, Elevation and QoS Comparison Chart

Figure 3 represents the analysis performed based on the proposed system comparing the capacitance, elevation and QoS of the mobile satellite service. This represents the energy efficiency of the system. The QoS is composed of exponential values for various elevation angles. This helps in analysing the power constraints provided by the system in $5 \mathrm{G}$ communication. Along with providing optimal performance and energy efficiency, this architecture also helps in improving the commercial value of the system.

\section{CONCLUSION AND FUTURE SCOPE}

This paper presents an improved architecture of mobile satellite systems that are integrated with 5G technology. It provides several benefits including high speed communication, portability, network access in all locations and during travel, ubiquity, energy efficiency and much more. The paper also presents expressions for optimization of power distribution and capacitance of the system. The provided simulation results confront the effectiveness of the system. These results can serve in the design of the $5 \mathrm{G}$ mobile satellite system. Future work involves improvising the algorithm to increase the efficiency rate. Further, specialized antennas are proposed to be developed to assist the communication purpose that can be integrated in the user system. 
J. Sustain. Wireless Syst.

Vol.01/ No. 03

Pages: 189-197

http://irojournals.com/irosws/

DOI: https://doi.org/10.36548/jsws.2019.3.005

\section{References}

[1] Liolis, Konstantinos, Alexander Geurtz, Ray Sperber, Detlef Schulz, Simon Watts, Georgia Poziopoulou, Barry Evans et al. "Use cases and scenarios of 5G integrated satellite-terrestrial networks for enhanced mobile broadband: The SaT5G approach." International Journal of Satellite Communications and Networking 37, no. 2 (2019): 91-112.

[2] Lee, Yonghwa, and Jihwan P. Choi. "Performance Evaluation of High-Frequency Mobile Satellite Communications." IEEE Access 7 (2019): 49077-49087.

[3] Khalili, Hamzeh, Pouria Sayyad Khodashenas, Carolina Fernandez, Daniel Guija, Konstantinos Liolis, Christos Politis, Gint Atkinson et al. "Benefits and Challenges of Software Defined Satellite-5G Communication." In 2019 15th Annual Conference on Wireless On-demand Network Systems and Services (WONS), pp. 1-4. IEEE, 2019.

[4] Evans, Barry G. "The role of satellites in 5G." In 2014 7th Advanced Satellite Multimedia Systems Conference and the 13th Signal Processing for Space Communications Workshop (ASMS/SPSC), pp. 197-202. IEEE, 2014.

[5] Vassaki, Stavroula, Athanasios D. Panagopoulos, and Philip Constantinou. "Effective capacity and optimal power allocation for mobile satellite systems and services." IEEE Communications Letters 16, no. 1 (2011): 6063.

[6] Wood, Peter. "Mobile satellite services for travellers." IEEE communications Magazine 29, no. 11 (1991): 3235.

[7] Jia, Min, Xuemai Gu, Qing Guo, Wei Xiang, and Naitong Zhang. "Broadband hybrid satellite-terrestrial communication systems based on cognitive radio toward 5G." IEEE Wireless Communications 23, no. 6 (2016): 96-106.

[8] Mak, Ka Ming, Hau Wah Lai, Kwai Man Luk, and Chi Hou Chan. "Circularly polarized patch antenna for future 5G mobile phones." IEEE Access 2 (2014): 1521-1529. 
J. Sustain. Wireless Syst.

Vol.01/ No. 03

Pages: 189-197

http://irojournals.com/irosws/

DOI: https://doi.org/10.36548/jsws.2019.3.005

[9] Zhang, Jiaxin, Xing Zhang, Muhammad Ali Imran, Barry Evans, Yan Zhang, and Wenbo Wang. "Energy efficient hybrid satellite terrestrial $5 \mathrm{G}$ networks with software defined features." Journal of Communications and Networks 19, no. 2 (2017): 147-161.

[10] Mahmoud, Korany R., and Ahmed M. Montaser. "Design of dual-band circularly polarised array antenna package for $5 \mathrm{G}$ mobile terminals with beam-steering capabilities." IET Microwaves, Antennas \& Propagation 12, no. 1 (2017): 29-39.

[11] Orsino, Antonino, Giuseppe Araniti, Pasquale Scopelliti, Irina A. Gudkova, Konstantin E. Samouylov, and Antonio Iera. "Optimal subgroup configuration for multicast services over 5G-satellite systems." In 2017 IEEE International Symposium on Broadband Multimedia Systems and Broadcasting (BMSB), pp. 1-6. IEEE, 2017.

[12] Gineste, Mathieu, Thibault Deleu, Michel Cohen, Nicolas Chuberre, Visvesh Saravanan, Valerio Frascolla, Markus Mueck, Emilio Calvanese Strinati, and Eryk Dutkiewicz. "Narrowband IoT service provision to 5G user equipment via a satellite component." In 2017 IEEE Globecom Workshops (GC Wkshps), pp. 1-4. IEEE, 2017.

[13] Chini, Paolo, Giovanni Giambene, and Sastri Kota. "A survey on mobile satellite systems." International Journal of Satellite Communications and Networking 28, no. 1 (2010): 29-57.

[14] Lunsford, J., R. Thorne, D. Gokhale, W. Garner, and G. Davies. "The AMSC/TMI mobile satellite services (MSS) system ground segment architecture." In 14th International Communication Satellite Systems Conference and Exhibit, p. 1855. 1992.

[15] Lamminen, H. "Mobile satellite systems." Journal of telemedicine and telecare 5, no. 2 (1999): 71-83.

[16] Li, Xichun, Abudulla Gani, Rosli Salleh, and Omar Zakaria. "The future of mobile wireless communication networks." In 2009 International Conference on Communication Software and Networks, pp. 554-557. IEEE, 2009.

[17] Yuan, Jun, Zhendong Li, Man Liu, Xinpeng Lv, and Ying Wang. "A Study on the Coexistence of TD-LTE/5G and Mobile Satellite Service." In 2018 24th Asia-Pacific Conference on Communications (APCC), pp. 119-124. IEEE, 2018.

ISSN: 2582-3167 (online) 
J. Sustain. Wireless Syst.

Vol.01/ No. 03

Pages: 189-197

http://irojournals.com/irosws/

DOI: https://doi.org/10.36548/jsws.2019.3.005

[18] Liu, Shuaijun, Bo Li, Gaofeng Cui, Xin Hu, and Weidong Wang. "Adaptive Interference Mitigation from IMT-2020 BS to Mobile-Satellite Service." In International Conference on Space Information Network, pp. 6678. Springer, Singapore, 2017.

[19] Saha, Rony Kumer. "A Hybrid System and Technique for Sharing Multiple Spectrums of Satellite Plus Mobile Systems With Indoor Small Cells in 5G and Beyond Era." IEEE Access 7 (2019): 77569-77596.

[20] Hassan, Syed Ali, Muhammad Shahmeer Omar, Muhammad Ali Imran, Junaid Qadir, and D. N. K. Jayako. "Universal access in 5G networks, potential challenges and opportunities for urban and rural environments." 5G Networks: Fundamental Requirements, Enabling Technologies, and Operations Management (2017). 